\title{
Molecular Biomarkers in Cytogenetically Normal - Acute Myeloid Leukemia: harnessing the targets
}

Monica Sharma $^{1 *}$ and Smita Mohanty ${ }^{2}$

${ }^{1}$ Assistant Professor, Department of Hematology, Safdarjang Hospital, New Delhi, India

${ }^{2}$ Associate Professor, Department of Medicine, Safdarjang Hospital, New Delhi, India

\begin{abstract}
Cytogenetics based risk adapted therapy has set the stage for personalized medicine for Acute Myeloid Leukemia but $50 \%$ of Acute Myeloid Leukemia cases exhibit normal cytogenetics. With the molecular techniques mutations like NPM1, CEBPA and FLT3-ITD have been discovered and are recommended by WHO and ELN. These are molecular prognostic biomarkers which have refined the risk stratification resulting in better but still very variable outcome and there is always a risk of over treating some cases using the conventional $3+7$ regimen. Thus there is a need to identify disease specific predictive biomarkers and translate them to targeted therapy. The implementation of high throughput molecular diagnostics has provided various biomarkers in the form of gene mutation and over expression, mi RNA and epigenetics which can be used for diagnosis, screening, monitoring, surveillance, or for providing predictive or prognostic information. Molecular biomarkers also play a role in development of targeted therapies. Some markers like FLT3-ITD have been identified against which specific inhibitors have been developed but many of these molecular lesions represent cooperating rather than initiating genetic events which cannot be targeted.
\end{abstract}

Eventually the cost and time to completion of whole-genome sequencing will decrease and it will be more convenient to sequence the entire genome from an individual patient rather than to screen for various mutations. Hopefully, in the future a ubiquitous target will be discovered that would be used across all the subgroups of Acute Myeloid Leukemia.

Keywords: CN-AML; Biomarkers; Prognostic markers; Predictive markers; Targeted therapy; Personalized medicine
Abbrevations: AML: Acute Myeloid Leukemia; CN- AML: Cytogenetic Ally Normal Acute Myeloid Leukemia; IR- AML: Intermediate Risk - Acute Myeloid Leukemia; NPM1: Nucleophosmin-1; CEBPA: CCAAT/Enhancer Binding Protein Alpha; FLT3-ITD: Fms-Like Tyrosine Kinase 3-Internal Tandem Duplication; WHO: World Health Organization; ELN: European Leukemia Net; CGH: Comparative Genome Hybridization; SNP: Single Nucleotide Polymorphism; mi RNA: Micro RNA; FAB: French American British; M3, M3v, and M4Eo: FAB categories; RT-PCR, qRT-PCR: Reverse Transcriptase Polymerase Chain Reaction,Quantitative RT-PCR; FISH: Florescence in situ Hybridization; MRD: Minimal Residual Disease; IDH1/2: Isocitrate Dehydrogenases1/2; DNMT3A: DNA methyltransferase 3A; TET2: tet oncogene family member 2, or Ten-Eleven Translocation; EZH2-: Enzymatic Component Of The Polycomb Repressive Complex; BCOR: BCL6 co repressor; allo HSCT, SCT: Allogenic Hematopoietic Stem Cell Transplant; MLL-PTD: Mixed Lineage Leukemia Partial Tandem Duplication; ASXL1: Additional Sex Combs Like 1; HDACi: Histone Deacetylase Inhibitors; ECOG E1900 trial: Eastern Cooperative Oncology Group; RAS, N RAS, K RAS: Rat Sarcoma/ Kirsten Ras Oncogene/ Neuroblastoma RAS Viral (v-ras) Oncogene Homolog; KIT: v-kit Hardy-Zuckerman 4 feline sarcoma viral oncogene homolog; WT1 mutation: Wilm's Tumor 1; RUNX1 mutations: Runt-Related Transcription Factor 1; MEK: Mitogen- Activated Protein Kinase Kinase-1; TP53 gene/P 53: Tumor Protein P53 Gene/ Protein 53; BAALC gene: Brain And Acute Leukemia, Cytoplasmic; MN1 gene: Meningioma 1; ERG- v: Ets Erythroblastosis Virus E26 Oncogene Homolog, Avian; EVI1: Ecotropic viral integration site 1; PI3/AKT/mTOR Pathways: Phosphatidylinositol-3-Kinase/ Akt, Also Known As Protein Kinase B/ Mammalian Target Of Rapamycin/ Phosphatase And Tensin Homologue Deleted On Chromosome Ten; NF-kB: Nuclear Factor Kappa From B Cells; Wnt/beta-catenin

pathways: Wingless-Related Integration Site; MDR1: Multi Drug Resistance Gene; P-gp: P Glycoprotein; LRP: Lung Resistance Protein; MRP1: MDR Associated Protein 1; Bcl-2: B-Cell Lymphoma 2; LSC: Leukemia Stem Cell; VEGF: Vascular Endothelial Growth Factor; CR: Complete Remission; EFS: Event Free Survival; RFS: Relapse Free Survival; OS: Overall Survival; DFS: Disease Free Survival; Ara-C: Cytosine Arabinoside

\section{Introduction}

Pretreatment cytogenetics is the backbone of current risk stratified but non specific treatment of Acute Myeloid Leukemia (AML). Nearly $50 \%$ of AML cases have no abnormality on karyotyping, even with comparative genomic hybridization (CGH) or SNP arrays [1-3]. Cytogenetically normal (CN-AML) patients, who comprise the largest subgroup and have been assigned an 'intermediate' prognosis, with five-year survival rates varying between $24 \%$ and $42 \%$ [ 4 ]. This clinical heterogeneity is related to the genomic heterogeneity of this cytogenetic subgroup in which several molecular aberrations have been identified.

Molecular characterization of CN-AML has been a major advancement in the management of AML patients. These mutations are the prognostic biomarkers and they influence treatment decisions

*Corresponding author: Monica Sharma, Assistant Professor Department of Hematology, Safdarjang Hospital, New Delhi, India, E-mail: monica_sharmamd@hotmail.com

Received April 15, 2013; Accepted June 17, 2013; Published June 19, 2013

Citation: Sharma M, Mohanty S (2013) Molecular Biomarkers in Cytogenetically Normal -Acute Myeloid Leukemia: harnessing the targets. J Mol Biomarkers Diagn S8:009. doi:10.4172/2155-9929.S8-009

Copyright: (c) 2013 Sharma M, et al. This is an open-access article distributed under the terms of the Creative Commons Attribution License, which permits unrestricted use, distribution, and reproduction in any medium, provided the original author and source are credited 
in terms of better prognostic stratification but lack predictability. Thus even when multitude of mutations have been discovered they have not been translated into effective targeted therapy and non specific $3+7$ regimen is still being used to treat this very heterogeneous category. There is always a risk of over treating many patients because of the lack of more specific drugs which require predictive biomarkers for their development.

The use of specific nontoxic drug All Trans Retinoic Acid (ATRA) in Acute Promyelocytic leukemia only proves that 'one drug fits all' practice should now give way to customized targeted therapy or personalized medicine. Personalized medicine has its underpinnings in the clinical molecular testing of diagnostic, prognostic, and predictive biomarkers.

Remarkable advancement in molecular diagnostics and highthroughput DNA sequencing has provided many biomarkers in the form of gene mutations and expressions, epigenetics and miRNA. There is a need to identify predictive biomarkers amongst them which can accurately predict the outcome in AML patients and can be matched with customized therapy based on individual prognostic risk and therapeutic response.

This review is an overview of the various genetic and epigenetic variations which have impact on the course of the disease along with the various available techniques and also some deterrents which have to be overcome for the genetic biomarkers to become standard of care.

\section{It is Important to Ensure that the Patient's Karyotype is Truly Normal}

Before proceeding for the molecular studies it is imperative to ensure that the karyotype of the CN-AML patient is truly normal. This is because rarely a CN AML case may harbor fusion genes due to cryptic recurrent translocations. To reliably pick up such a case it is recommended that analysis of at least 20 metaphases from a marrow sample cultured in vitro for 24 to 48 hours is performed [5-7].

Such an investigation is warranted especially in CN-AML patients with $\mathrm{FAB}$ categories $\mathrm{M} 3, \mathrm{M} 3 \mathrm{v}$, and M4Eo morphology but is otherwise not routinely recommended outside of a clinical trial [8]. The RT-PCR and FISH can detect the cryptic recurrent translocations while spectral karyotyping [9], FISH with a comprehensive set of genomic DNA probes [10], and comparative genomic hybridization [11] can confirm the absence of any other unrecognized chromosome aberrations.

\section{Various Categories of Mutations with its Respective Targeted Therapy}

\section{Mutations with widely recognized clinical impact}

Molecular markers that have been incorporated in WHO and ELN classification systems are [12] are NPM1, FLT3-ITD and CEBPA. They are recommended to be used in routine in order to identify patients who would benefit from allo SCT. The FLT3-ITD is associated with aggressive disease and poor outcome while biallelic CEBPA mutation and NPM1 without FLT3-ITD have favorable outcome. The FLT3ITD and NPM1 mutations frequently coexist and their interaction influences the prognosis hence both should be tested upfront in all patients with CN-AML and when both NPM1 and FLT3-ITD mutations are negative, CEBPA mutations should be tested for further risk stratification.

\section{Targeted therapy}

CN-AML patients harboring CEBPA and particularly NPM1 mutations without FLT3-ITD mutations are sensitive to the induction therapy [13] and standard induction chemotherapy followed by three to four cycles of high-dose cytarabine is recommended in younger adults ( $<60$ years old) with CN-AML. No targeted therapy so far exists for the two favorable mutations but Blasts with NPM1 mutations exhibit high density of CD33 and addition of anti-CD33 immunoconjugate to chemotherapy has been tried [14]. Also ATRA with induction therapy has been tried in elderly CN-AML expressing NPM1 mutations [15]. Small molecules which can interfere with the oligomerization properties of NPM1 have shown anti-leukemic activity in vitro and may enter in clinical trial [16]. Thus CEBPA and particularly NPM1 mutations are only prognostic biomarkers the only predictive marker in use currently is the FLT3-ITD mutation against which multi-targeted TKI are in early phases of clinical development viz sorafenib, lestaurtinib, midostaurin, quizartinib and tandutinib. All exert some anti-leukemic activity, but when used as single agents have been disappointing hence FLT3 inhibitors have been tried combined with chemotherapy, in Phase III trials $[17,18]$. FLT3 mutation because of its mutability is not good target for MRD [19] but it is expressed on the LSC and FLT3 inhibitors are being tried [20].

\section{Epigenetic modifier genes}

A significant class of the new mutations is epigenetic modifier genes which include the IDH1, IDH2, DNMT3A and TET2 genes. With the exception of TET2 mutations, all other mutations have been identified by massively parallel sequencing.

\section{IDH1 and IDH2 mutations}

Mutations in IDH1 and IDH2 occur in 10-12\% CN-AML patients [21]. Mutant IDH enzymatic activity in AML converts alpha ketoglutarate to 2 hydroxyglutarate (2-HG) which is the oncometabolite associated with leukemogenesis. Raised 2-HG can be detected in the serum and BM of AML patients with IDH1/2 mutations and serves as a biomarker for this group of patients and also as a measure of minimal residual disease $[22,23]$. There is a mutual exclusivity between IDH1/2 and TET2 mutations in AML and significant co occurrence with NPM1 mutations [24]. Mutations in the IDH1 occur at R132 while at IDH2 at R172. Where mutations at IDHR172 do not correlate with outcome or response to therapy, IDHR132 mutation had worse outcome in patients with the FLT3 wild type genotype, in contrast IDH R140Q mutation conferred improved OS [25].

\section{DNMT3A mutations}

DNMT3A mutations have been identified in $20 \%$ acute myeloid leukemia (AML). It is the second most common somatic mutation [21] after FLT3 ITD mutations, within CN-AML.The DNMT3A R882H mutations is associated with NPM1, FLT3-ITD and IDH1 mutations and is also with BCOR mutations.

DNMT3A mutations can identify new subgroups of CN-AML patients with shorter overall and disease free survival. In fact, DNMT3A mutations appear to exert their negative impact mostly in the highrisk category of CN-AML (wild-type NPM1 and wild-type FLT3), for which more intensive treatments, including allo HSCT, are already recommended [26-28].

\section{TET2 mutations}

The Tet oncogene family member 2 (TET2) encodes for proteins that are involved in epigenetic regulation. TET2 mutations have been detected in $7.6 \%$ of AML and also in association with CN-AML. The 
enzymatic activity of TET2 is impaired by $2-\mathrm{HG}$ produced by mutant IDH1/2 hence mutations of IDH1/2 are mutually exclusive with mutations of TET2 $[18,29]$. The prognostic impact of TET2 mutations in AML is unclear [18].

\section{MLL mutations}

Partial Tandem Duplication (PTD) of MLL has been detected in $5-11 \%$ of CN-AML $[4,24]$. It is proposed to contribute to AML development through DNA hypermethylation and epigenetic silencing of tumor suppressor genes [30]. MLL-PTD may be associated with FLT3-ITD and FLT3 point mutations and have shortened remission duration and shorter Disease-Free Survival (DFS) [31].

\section{ASXL1 mutations}

ASXL1 mutations are much more common in elderly than younger AML patients (16\% vs. $3-5 \%)$ [18]. Although rare in AML ASXL1 mutation is associated with adverse overall survival [21].

\section{Targeted therapy}

Unlike chromosomal deletions where there is an irreversible loss of function, epigenetic mutations can be reversed using HDACi and hypomethylating agents like 5-aza-2'-deoxycytidine (decitabine) and 5-azacytidine [32].

No specific targeted therapy apart from the hypomethylation therapy is available for this subset of AML. A small molecule inhibitor has been developed which would block the synthesis of 2-HG and potentially be the specific drug for IDH1/2 mutations [33,34]. For MLL mutations a specific therapy has been developed to target DOT1L, which is a histone methyltransferase utilized by the rearranged/ mutated MLL. Recently small molecule inhibitor of DOT1L namely EPZ-5676 has entered human clinical trial [35].

ECOG E1900 trial was conducted using several of the above markers and survival was seen to be improved with escalated dose of daunorubicin among patients with DNMT3A or NPM1 mutations or MLL translocations [36]. Using gene mutations to segregate patients who may benefit from higher doses of chemotherapy was the step in the direction of personalized therapy for AML.

The mutations cooperate in the leukemogenesis and understanding of which mutations occur together can provide important prognostic information. More extensive mutational analysis differentiates otherwise 'unremarkable on cytogenetics' AML category into clinically relevant risk groups [37]. FLT3-ITD has been shown to be an independent prognostic factor for poor outcome in AML in many studies hence using it as the starting point the following risk stratification has been proposed for CN-AML [21].

\section{FLT3-ITD-negative}

1. NPM1/IDH mutant patients represent a favorable-risk AML.

2. NPM1 mutant patients without concurrent IDH mutations have less favorable outcome.

3. Presence of TET2, ASXL1, and/or MLL-PTD is associated with very adverse overall survival.

\section{FLT3-ITD mutant}

1. Concurrent mutations in TET2, DNMT3A, MLL-PTD, forms a very-high-risk subset which may impact the treatment decisions. Large number (approximately 47\%) of the total intermediate-risk cases fall into this category which harbor any one of the above four mutations.

2. In the absence of the above 4 genetic abnormalities outcome is same as that of FLT3-ITD/CEBPa double-mutant patients.

This mutationally defined risk stratification would be clinically significant to identify patients with favorable risk who would have better outcome than even core binding factor-positive AML cases simply with standard induction and consolidation. Patients with adverse-risk AML fare badly with standard therapy and require SCT or investigational therapy for cure [21].

In a large study [38] using multiplexed mass spectrometry, 344 mutations across 31 genes were screened which are associated with leukemia. It was observed that between the normal and abnormal cytogenetic risk groups, the mutation frequency and distribution is very different, suggesting different biology. Not only was the mutation frequency in CN AML at 78\%, twice that seen in abnormal cytogenetics. The mutation distribution was also distinct which included more frequent IDH1, NPM1, and NPM1-FLT3 overlapping mutations in the normal cytogenetic group and increased RAS, KIT, and isolated FLT3-ITD mutations in the abnormal cytogenetic group. Concurrent mutations in 2 or more genes were seen almost exclusively in patients with normal cytogenetics In the CN-AML cases, FLT3, NPM1, IDH1, and CEBPA mutations were seen more commonly as multiple mutations than in isolation [38]. Such mutational profiling not only helps to understand the biology and prognosticate the patients it may also help selecting patients for clinical trials with novel inhibitors.

\section{Other Mutations}

Apart from the above mutations there are some newer less common ones which also influence the prognosis.

\section{WT1 gene}

These mutations are detectable in 10-13\% of CN-AML [39-43] and in multivariable analysis, WT1mutation was an independent adverse prognostic factor and the WT1mut/FLT3-ITD ${ }^{\text {pos }}$ genotype appeared to be associated with worse clinical course. Gaidzik et al. suggested that the negative impact ofWT1 mutations as reported by others may be overcome by the use of repetitive cycles of high-dose cytarabine [41]. SNP rs16754 located in the mutational hot spot of WT1 in exon 7 has been shown to be associated with favorable outcome in patients with CN-AML and increased chemo sensitivity [44].

\section{RUNX1 gene}

Mutations usually cluster in the Runt domain of the gene. Frequencies of RUNX1 mutations within CN-AML are variable, ranging from $6.3 \%$ [45] to $26.3 \%$ [46]. The Mutations are associated with resistance to chemotherapy and inferior EFS, RFS, and OS [4547]; however an improved RFS is seen with allo HSCT.

\section{BCOR (BCL6 co repressor) gene}

Mutations of the BCOR gene were discovered by whole exome sequencing of a single CN-AML patient [48]. Since then has been found to occur in about $4 \%$ of all CN-AML and occur with DNMT3A mutations. They predict a poorer prognosis [48] and act by interfering with epigenetic mechanisms [49].

\section{RAS gene}

NRAS mutation in CN-AML has been found in 9\% adult and 14\% younger 60 years, patients [50]. Although NRAS mutations have been 
known to have no prognostic impact they provide a target for molecular therapy [33]. More recently, Neubauer et al. showed a predictive impact of RAS mutations in that patients receiving high-dose cytarabine in consolidation therapy had a significantly lower probability of relapse as compared with patients receiving standard dose cytarabine [51]. RAS mutations also render the cells sensitive to MEK inhibitors and currently clinical trials investigating MEK inhibitors in myeloid malignancies with NRAS and/or KRAS mutations are underway [52].

\section{TP53 gene}

P53 gene mutations are found in less than $10 \%$ of de novo AML and are associated with older age, resistance to chemotherapy and very short survival [53].

\section{Over Expression of Genes}

\section{The BAALC (brain and acute leukemia, cytoplasmic) gene}

High BAALC expression levels predict lower CR rates and also low disease-free and overall survival (OS) [42,54].

\section{MN1 (meningioma 1) gene}

MN1 over expression is associated with poor response to induction chemotherapy higher relapse rate and worse OS. While low MN1 expression has been correlated with response to ATRA in elderly non APML patients, thereby suggesting that MN1 expression is not only a prognostic but also a predictive marker for response to treatment. In younger than 60 years CN-AML cases, higher MN1 expression is associated with NPM1 wild-type status and increased BAALC expression $[42,55]$.

\section{ERG (v-ets erythroblastosis virus E26 oncogene homolog,} avian)

The adverse prognostic significance of over expression of ERG has been established in CN-AML [56]. High ERG expression levels influences outcome of low molecular risk CN-AML (mutated NPM1 without FLT3-ITD). In a GEP study ERG expression was the strongest negative prognostic factor and provided prognostic information in addition to established parameters (eg, FLT3-ITD) [42].

\section{EVI1 (ecotropic viral integration site 1)}

High EVI1 expression predicts poor outcome in the cytogenetic intermediate-risk group. Patients with high EVI1 expression had significantly better 5 -year relapse-free survival and OS who received allo HSCT in the first CR $[55,57]$.

\section{EZH2 mutations}

Recently found in a male with CN-AML [58]. Over expression of EZH2 has been reported in both solid tumors and blood cancers $[59,60]$.

\section{Targeted therapy}

Recently an S-adenosyl homocysteine hydrolase inhibitor named 3-Deazaneplanocin A (DZNep) has been shown to induce selective apoptosis in cancer cells sparing the normal cells and removed EZH2 [61-64]. This compound has not reached yet the clinical trial setting. Apart from this the above markers are investigational and so far have had no bearing on treatment decisions but are important prognostic markers. For many of them, there are still not enough studies evaluating their prognostic and predictive impact.

\section{Other Molecular Biomarkers}

\section{Micro RNA (miR)}

Recent studies have also shown that changes in microRNA expression can affect clinical outcome in AML. Garzon et al. [65] reported that, across all cytogenetic subgroups, over expressed miR20a, miR-25, miR-191, miR-199a, and miR-199b adversely affected OS. Using 12 micro RNA probes an expression signature which associated with event-free survival has been defined in patients with CN-AML belonging to the molecular high-risk group (FLT3-ITD ${ }^{\text {pos }}+$ NPM1 wildtype). Five probes in the signature represented miR-181a and miR-181b; down regulation of the members of miR-181 family attributed to the aggressive leukemia phenotype and high levels of miR-181 expression is associated with less aggressive disease and predicts favorable outcome in CN-AML [64].

\section{Therapeutic implications}

Synthetic oligonucleotides targeting specific miRNAs have been proposed as a therapy with potential anti-leukemic activity.

\section{PI3K/AKT/mTOR pathways}

Most AML cases show activated PI3K/AKT/mTOR pathways [6567]. PI3 kinase inhibitors such as LY294002 and PI-103, have shown to induce apoptosis in AML cells ex vivo [68,69]. Inhibition of Akt with perfosine [70] when combined with MEK inhibitors in preclinical studies has shown kill AML cells ex vivo [71]. Although rapamycin, an mTORC1 inhibitor, failed to show responses in clinical trial as a single agent [72] it may have potentially have some role as a chemo sensitizing agent [73]. PI3K and mTORC1/2 inhibitors like temsirolimus when combined with chemotherapy like clofarabine in elderly high risk AML has been observed to achieve much better clinical responses than either agent alone [74-76]. mTOR activation which is the downstream of PI3K and AKT results in the phosphorylation ribosomal S6 protein which is a robust marker and can be noted by flow cytometry and can be used as a molecular marker to develop drugs that would target this pathway [77].

\section{Response to Therapy}

\section{Minimal residual disease (MRD)}

The MRD is an in vivo measure of drug sensitivity and provides a patient-specific parameter predictive of risk of relapse. The newly discovered molecular prognostic biomarkers are being tested for their viability to be used for MRD measurement. For this purpose, it is important to choose a marker that is measureable and stable throughout the disease course. An NPM1 mutation has been proved to be a suitable biomarker since it remains stable throughout the course of the disease including relapse [78].

Increased level of MRD is an indication for either allogeneic transplantation or other investigational therapies. But MRD to be clinically relevant must be used along with pretreatment variables to designate patients into specific risk categories [19].

\section{Multidrug resistance}

It is the levels of multidrug resistance gene 1 (MDR1) encoding P-glycoprotein (Pgp) as well as the MDR associated protein 1 (MRP1) and lung resistance protein (LRP) which mediate drug resistance and correlate with response to chemotherapy and clinical outcome.

Various studies have evaluated the prognostic significance of 
expression of MDR genes [42,79-81]. Early studies demonstrated significant association between expression of MDR genes and clinical outcome [82-84], while the later studies [85] demonstrated that though MDR genes may not be an independent prognostic factor, but it can be a potential therapeutic target. Several agents have been developed to impair the function of proteins encoded by MDR genes, [86-89] and in combination with conventional chemotherapy, these agents sensitize the cells to the chemotherapy and thus improve clinical outcome [89].

Evasion of apoptosis is also connected to drug resistance. Bcl-2 is an apoptosis inhibitor protein when over expressed renders tumor cells resistant to induction of apoptosis. As high levels of bcl-2, in AML has been pursued as a potential drug target using agents such as antisense oligonucleotides and small molecular inhibitors [90-92]. Another strategy to induce apoptosis is by the novel compound APR-246, which restores the function of $\mathrm{p} 53$ which has been tested in a clinical trial in patients with hematological malignancies [93]

\section{The Leukemic Stem Cells (LSCs)}

LSCs represent a reservoir of leukemia and current treatment is cytotoxic to the tumor bulk, but not to the leukemic stem cells that culminate into relapse and resistance to chemotherapy [94,95]. The signaling pathways like NF-kB, PI3K/Akt/mTOR, and Wnt/betacatenin pathways are important for the survival of LSCs and have obvious therapeutic implications [96]. Parthenolide has been found to induce apoptosis in AML; it is a potent inhibitor of NF-kB, and compared to cytarabine much more selectively eliminates LSCs thus sparing normal hematopoietic cells [97]. The PI3/AKT/mTOR pathway, constitutively activated in most AMLs, is important for the LSCs and is being targeted by mTOR inhibitors, three of which rapamycin, temsirolimus, and everolimus, are undergoing clinical trials [72,73]. Pharmacologic inhibition of activated phosphatidylinositide-3 kinase by LY294002 is also being studied which would survival of LSCs [98]. Relationship of LSC with its microenvironment is the new target for drug development. Antiangiogenic drugs $[99,100]$ are the main drug of interest. Trials of angiogenesis inhibitors (angiostatin, endostatin, and platelet factor-4), antibodies to VEGF or fibroblast growth factor, or inhibitors of endothelial cell proliferation, such as SU5416, are underway [101]. Agents such as granulocyte colony stimulating factor cause LSCs to cycle, disrupting them from their vascular niche and increased their sensitivity to cytarabine [102]. Rakicidin A is another compound which may target a hypoxic microenvironment [103]. Several FLT3 inhibitors, including CEP701and PKC412, are also being tried in phase II clinical trials in AML targeting the markers on the LSC [104].

Cyclosporine A which is a P-glycoprotein inhibitor when used along with standard cytotoxic chemotherapy was found to improve clinical outcomes in poor risk patients with AML [105]. However, studies with another P-glycoprotein inhibitor valspodar (PSC-833) failed to replicate the results [106]. Currently P-glycoprotein inhibitor zosuquidar is undergoing clinical trial in combination with standard cytotoxic agents. Breast cancer resistance protein inhibitors are much more fastidious and effective, but are still in nascent stage of clinical development $[107,108]$. The differential expression of miRNAs on the LSCs is being targeted for the eradication of LSCs $[109,110]$

\section{Pharmacogenomics}

It is the study of how genetic variants influence drug efficacy and toxicity. By sequencing the germline and leukemia genomes in patients with AML, it would be possible to identify genetic variants known to affect the efficacy and toxicity of chemotherapeutic agents which subsequently influence the choice and dose of drugs used [111]. The pharmacogenetics testing can be implemented even before the treatment begins. Pharmacogenomics thus makes it possible to optimize the dosage of existing therapeutics as has been studied with Ara-C [112]. Detection of various polymorphisms in genes involved in metabolism using the newer high-throughput genomics will increase in the utility of pharmacogenomics.

\section{Molecular Biomarker Detection Methodologies}

Although karyotyping is the basis for classification in 2008 World Health Organization classification scheme and it is possible to categorize over two-thirds of AML compared to only one-third in the 2001 World Health Organization scheme. But it was the molecular technologies which has catapulted the categorization of the otherwise cytogenetically unremarkable intermediate risk CNAML cases into risk adapted categories thus allowing not only for better understanding of leukemogenesis but also biomarker discovery and potential for targeted therapy.

The implementation of DNA and protein microarrays has accelerated the biomarker discovery like never before. The ultimate goal is to identify sets of disease specific biomarkers and then to combine them with a robust screening technology. Though this technique is not yet applicable for MRD detection for which PCR is preferred. It can also be an alternative to microarray- especially when genes in very low copy number have to be confirmed. Quantitative RT-PCR (qRT-PCR), where signals are simultaneously generated in an exponential way is the most sensitive technique for mRNA detection and quantitation [113]. Hundreds of SNPs across the whole genome can be analyzed with the help of SNPs arrays. DNA copy number, loss of heterozygosity and chromosomal modifications can also be picked up using array technology. Gene expression profiling based on array technology not only identifies potential disease biomarkers but also improves the understanding of the molecular biology of the disease.

Although automated sequencing instruments (sequencers) allow high-throughput nucleotide sequencing full-genome sequencing and exonic sequencing is now feasible with rapidly decreasing turnover time and may be in near future available for clinical use to identify druggable targets and markers for monitoring tumor burden. Unique subtypes of AML have been identified on the basis of DNA methylation signatures, which have been found to be predictive of survival but not yet of response to hypomethylating agents [114].

Newer high throughput technologies have made it possible to categorize $80 \%$ of AML cases based on molecular biomarkers to predict outcome and select appropriate treatment but more than $25 \%$ of AML patients still remain uncategorized which exhibit no mutations in the known leukemia-associated genes [115]. More sophisticated technology needs to be developed for these patients.

\section{Deterrents in the Development of Targeted Therapy}

Biomarker-based drug development is a proven strategy in the development of new drugs. The implementation of DNA and protein microarrays has accelerated the biomarker discovery. The ultimate goal is to identify sets of disease specific biomarkers which would allow stratification based on clinical molecular testing thus evading the toxic effects and morbidity by patient selection.

Despite the rapid developments of molecular technologies and 
newer biomarkers being discovered it has not translated into the targeted drug development. This has been marred by many problems.

1. Due to their relative infrequency of the mutations conclusions about their significance cannot be made with great confidence.

2. Genetic complexity of AML is characterized by several coexisting mutations hence interactions with other molecular markers would be important but were not taken into account.

3. Their mere discovery offers potential targets for novel therapies, but to identify the truly 'druggable' ones would require delineating the driver mutations where most of the mutations which have been identified are passenger mutations.

4. The prognostic impact of the markers has been mostly evaluated in retrospective studies, involving low patient numbers.

5. Turnaround time for their results is still not appropriate to plan the induction regimen based on the genetic profile of patients.

6. The cancer biomarkers in clinical use are not suitable for population screening or for early diagnosis.

7. Prohibitive cost of genotyping or sequencing deters the progress of biomarker discovery and utilization.

8. Targeting essential cell functions creates drugs with narrow therapeutic indices. These new agents are unlikely to be curative when administered as monotherapy. Thus, new compounds with an antileukemic activity need to be tested in combination with other new agents or with already approved conventional drugs.

9. Drugs targeting the regulators of cellular proliferation, survival, angiogenicity or immunogenicity of leukemic cells may produce collateral damage in normal cells which share these properties.

Large prospective multicentric studies perhaps on international scale are clearly warranted to clarify these caveats.

\section{Conclusion}

In conclusion, the most immediate application of molecular prognostic markers is in the decision whether or not to proceed to SCT in first CR based on a risk-adapted approach. The molecular biomarkers also have immense role in the design and implementation of targeted therapies which is making its way from disease-based therapeutic regimens to molecular target-based protocols. Hopefully a more ubiquitous 'fit for purpose' biomarker will be discovered that can be used across the AML subgroups and there will not be a requirement for a unique therapy matched for each subgroup.

\section{References}

1. Bullinger L, Kronke J, Schon C, Radtke I, Urlbauer K, et al. (2010) Identification of acquired copy number alterations and uniparental disomies in cytogenetically normal acute myeloid leukemia using high-resolution single-nucleotide polymorphism analysis. Leukemia 24: 438-449

2. Suela J, Alvarez S, Cigudosa JC (2007) DNA profiling by arrayCGH in acute myeloid leukemia and myelodysplastic syndromes. Cytogenet Genome Res 118: 304-309.

3. Walter MJ, Shen D, Ding L, Shao J, Koboldt DC, et al. (2012) Clonal architecture of secondary acute myeloid leukemia. N Engl J Med 366: 1090-1098.

4. Mro'zek K, Marcucci G, Paschka P, Whitman SP, Bloomfield CD, et al. (2007) Clinical relevance of mutations and gene-expression changes in adult acute myeloid leukemia with normal cytogenetics: Are we ready for a prognostically prioritized molecular classification? Blood 109: 431- 448.
5. Rowe D, Cotterill SJ, Ross FM, Bunyan DJ, Vickers SJ, et al. (2000) Cytogenetically cryptic AML1-ETO and CBF beta-MYH11 gene rearrangements: incidence in 412 cases of acute myeloid leukaemia. $\mathrm{Br} \mathrm{J}$ Haematol 111: 1051 1056.

6. Grimwade D, Biondi A, Mozziconacci MJ, Hagemeijer A, Berger R, et al. (2000) Characterization of acute promyelocytic leukemia cases lacking the classic t(15;17): results of the European Working Party. Groupe Français de Cytogénétique Hématologique, Groupe de Français d'Hematologie Cellulaire, UK Cancer Cytogenetics Group and BIOMED 1 European Community Concerted Action "Molecular Cytogenetic Diagnosis in Haematological Malignancies". Blood 96: 1297-1308.

7. Gamerdinger U, Teigler-Schlegel A, Pils S, Bruch J, Viehmann S, et al. (2003) Cryptic chromosomal aberrations leading to an AML1/ETO rearrangement are frequently caused by small insertions. Genes Chromosomes Cancer 36: $261-$ 272.

8. National Comprehensive Cancer Network. Clinical Practice Guidelines in Oncology-v.2.2011: Acute Myeloid Leukemia.

9. Preiss BS, Kerndrup GB, Pedersen RK, Hasle H, Pallisgaard N. (2006) Contribution of multiparameter genetic analysis to the detection of genetic alterations in hematologic neoplasia: an evaluation of combining G-band analysis, spectral karyotyping, and multiplex reverse-transcription polymerase chain reaction (multiplex RT-PCR). Cancer Genet Cytogenet 165: 1-8.

10. Cuneo A, Bigoni R, Cavazzini F, Bardi A, Roberti MG, et al. (2002) Incidence and significance of cryptic chromosome aberrations detected by fluorescence in situ hybridization in acute myeloid leukemia with normal karyotype. Leukemia 16: $1745-1751$.

11. Casas S, Aventín A, Fuentes F, Vallespí T, Granada I, et al. (2004) Genetic diagnosis by comparative genomic hybridization in adult de novo acute myelocytic leukemia. Cancer Genet Cytogenet 153: 16-25.

12. Döhner $\mathrm{H}$, Estey $\mathrm{EH}$, Amadori $\mathrm{S}$, Appelbaum $\mathrm{FR}$, Büchner $\mathrm{T}$, et (2010) Diagnosis and management of acute myeloid leukemia in adults: recommendations from an international expert panel, on behalf of the European LeukemiaNet. Blood 115: 453-474.

13. Schneider F, Hoster E, Unterhalt M, Schneider S, Dufour A, et al. (2009) NPM1 but not FLT3-ITD mutations predict early blast cell clearance and CR rate in patients with normal karyotype AML (NK-AML) or high-risk myelodysplastic syndrome (MDS). Blood 113: 5250-5253.

14. Pogosova-Agadjanyan E, Kopecky K, Petersdorf S, Erba HP, Stone R, et al. (2011) Prognostic significance of FLT3 and NPM1 mutations in adults of age 18-60 with De Novo Acute Myeloid Leukemia (AML) on SWOG S0106 study: a study by FHCRC and SWOG. Blood 2520.

15. Schlenk RF, Döhner K, Kneba M, Götze K, Hartmann F, et al. (2009) Gene mutations and response to treatment with all-trans retinoic acid in elderly patients with acute myeloid leukemia. Results from the AMLSG Trial AML HD98B. Haematologica 94: 54-60.

16. Balusu R, Fiskus W, Rao R, Chong DG, Nalluri S, et al. (2011) Targeting levels or oligomerization of nucleophosmin 1 induces differentiation and loss of survival of human AML cells with mutant NPM1. Blood 118: 3096-3106.

17. Fathi A, Levis M (2011) FLT3 inhibitors: a story of the old and the new. Cur Opin Hematol 18: 71-76.

18. Martelli MP, Sportoletti P, Tiacci E, Martelli MF, Falini B (2013) Mutationa landscape of AML with normal cytogenetics: biological and clinical implications. Blood Rev 27: 13-22.

19. Buccisano F, Maurillo L, Del Principe MI, Del Poeta G, Sconocchia G, et al. (2012) Prognostic and therapeutic implications of minimal residual disease detection in acute myeloid leukemia. Blood 119: 332-341.

20. Levis M, Murphy KM, Pham R, Kim KT, Stine A, et al. (2005) Internal tandem duplications of the FLT3 gene are present in leukemia stem cells. Blood 106 673-680.

21. Patel JP, Levine RL (2012) How do novel molecular genetic markers influence treatment decisions in acute myeloid leukemia? Hematology Am Soc Hematol Educ Program 2012: 28-34.

22. Ward PS, Patel J, Wise DR, Abdel-Wahab O, Bennett BD, et al. (2010) The common feature of leukemia-associated IDH1 and IDH2 mutations is a neomorphic enzyme activity converting alpha-ketoglutarate to 2-hydroxyglutarate. Cancer Cell 17: 225-234.

23. Gross S, Cairns RA, Minden MD, Driggers EM, Bittinger MA, et al. (2010) 
Citation: Sharma M, Mohanty S (2013) Molecular Biomarkers in Cytogenetically Normal -Acute Myeloid Leukemia: harnessing the targets. J Mol Biomarkers Diagn S8:009. doi:10.4172/2155-9929.S8-009

Cancer-associated metabolite 2-hydroxyglutarate accumulates in acute myelogenous leukemia with isocitrate dehydrogenase 1 and 2 mutations. J Exp Med 207: 339-344

24. Döhner H, Gaidzik VI (2011) Impact of genetic features on treatment decisions in AML. Hematology Am Soc Hematol Educ Program 2011: 36-42.

25. Green CL, Evans CM, Zhao L, Hills RK, Burnett AK, et al. (2011) The prognostic significance of IDH2 mutations in AML depends on the location of the mutation. Blood 118: 409-412.

26. Ley TJ, Ding L, Walter MJ, McLellan MD, Lamprecht T, et al. (2010) DNMT3A mutations in acute myeloid leukemia. N Engl J Med 363: 2424-2433.

27. Hou HA, Kuo YY, Liu CY, Chou WC, Lee MC, et al. (2012) DNMT3A mutations in acute myeloid leukemia: stability during disease evolution and clinical implications. Blood 119: 559-568.

28. Thol F, Damm F, Lüdeking A, Winschel C, Wagner K, et al. (2011) Incidence and prognostic influence of DNMT3A mutations in acute myeloid leukemia. $J$ Clin Oncol 29: 2889-2896.

29. Figueroa ME, Abdel-Wahab O, Lu C, Ward PS, Patel J, et al. (2010) Leukemic IDH1 and IDH2 mutations result in a hypermethylation phenotype, disrupt TET2 function, and impair hematopoietic differentiation. Cancer Cell 18: 553-567.

30. Whitman SP, Hackanson B, Liyanarachchi S, Liu S, Rush LJ, et al. (2008) DNA hypermethylation and epigenetic silencing of the tumor suppressor gene, SLC5A8, in acute myeloid leukemia with the MLL partial tandem duplication. Blood 112: 2013-2016.

31. Schlenk RF, Döhner K (2009) Impact of new prognostic markers in treatment decisions in acute myeloid leukemia. Curr Opin Hematol 16: 98-104

32. Oki Y, Aoki E, Issa JP (2007) Decitabine--bedside to bench. Crit Rev Onco Hematol 61: 140-152.

33. Akalin A, Garrett-Bakelman FE, Kormaksson M, Busuttil J, Zhang L, et al (2012) Base-pair resolution DNA methylation sequencing reveals profoundly divergent epigenetic landscapes in acute myeloid leukemia. PLoS Genet 8 : e1002781.

34. Yen KE, Bittinger MA, Su SM, Fantin VR (2010) Cancer-associated IDH mutations: biomarker and therapeutic opportunities. Oncogene 29: 6409-6417.

35. Daigle SR, Olhava EJ, Therkelsen CA, Majer CR, Sneeringer CJ, et al. (2011) Selective killing of mixed lineage leukemia cells by a potent small-molecule DOT1L inhibitor. Cancer Cell 20: 53-65.

36. Lee JH, Joo YD, Kim H, Bae SH, Kim MK, et al. (2011) A randomized tria comparing standard versus high-dose daunorubicin induction in patients with acute myeloid leukemia. Blood 118: 3832-3841.

37. Patel JP, Gönen M, Figueroa ME, Fernandez H, Sun Z, et al. (2012) Prognostic relevance of integrated genetic profiling in acute myeloid leukemia. $N$ Engl J Med 366: 1079-1089.

38. Dunlap J, Beadling C, Warrick A, Neff T, Fleming WH, et al. (2012) Multiplex high-throughput gene mutation analysis in acute myeloid leukemia. Hum Pathol 43: $2167-2176$

39. Paschka P, Marcucci G, Ruppert AS, Whitman SP, Mrózek K, et al. (2008) Wilms' tumor 1 gene mutations independently predict poor outcome in adults with cytogenetically normal acute myeloid leukemia: a cancer and leukemia group B study. J Clin Oncol 26: 4595-4602.

40. Virappane P, Gale R, Hills R, Kakkas I, Summers K, et al. (2008) Mutation of the Wilms' tumor 1 gene is a poor prognostic factor associated with chemotherapy resistance in normal karyotype acute myeloid leukemia: the United Kingdom Medical Research Council Adult Leukemia Working Party. J Clin Oncol 26 5429-5435.

41. Gaidzik VI, Schlenk RF, Moschny S, Becker A, Bullinger L, et al. (2009) Prognostic impact of WT1 mutations in cytogenetically normal acute myeloid leukemia: a study of the German-Austrian AML Study Group. Blood 113: 45054511.

42. Marcucci G, Haferlach T, Döhner H (2011) Molecular genetics of adult acute myeloid leukemia: prognostic and therapeutic implications. J Clin Oncol 29: 475-486.

43. Owen C, Fitzgibbon J, Paschka P (2010) The clinical relevance of Wilms Tumour 1 (WT1) gene mutations in acute leukaemia. Hematol Oncol 28: 13-19.

44. Damm F, Heuser M, Morgan M, Yun H, Grosshennig A, et al. (2010) Single nucleotide polymorphism in the mutational hotspot of WT1 predicts a favorable outcome in patients with cytogenetically normal acute myeloid leukemia. J Clin Oncol 28: 578-585.

45. Gaidzik VI, Bullinger L, Schlenk RF, Zimmermann AS, Röck J, et al. (2011) RUNX1 mutations in acute myeloid leukemia: results from a comprehensive genetic and clinical analysis from the AML study group. J Clin Oncol 29: 13641372.

46. Schnittger S, Dicker F, Kern W, Wendland N, Sundermann J, et al. (2011) RUNX1 mutations are frequent in de novo AML with noncomplex karyotype and confer an unfavorable prognosis. Blood 117: 2348-2357.

47. Tang JL, Hou HA, Chen CY, Liu CY, Chou WC, et al. (2009) AML1/RUNX mutations in 470 adult patients with de novo acute myeloid leukemia: prognostic implication and interaction with other gene alterations. Blood 114: 5352-5361.

48. Grossmann V, Tiacci E, Holmes AB, Kohlmann A, Martelli MP, et al. (2011) Whole-exome sequencing identifies somatic mutations of BCOR in acute myeloid leukemia with normal karyotype. Blood 118: 6153-6163.

49. Tiacci E, Grossmann V, Martelli MP, Kohlmann A, Haferlach T, et al. (2012) The corepressors BCOR and BCORL1: two novel players in acute myeloid leukemia. Haematologica 97: 3-5

50. Estey E, Döhner H (2006) Acute myeloid leukaemia. Lancet 368: 1894-1907.

51. Neubauer A, Maharry K, Mrózek K, Thiede C, Marcucci G, et al. (2008) Patients with acute myeloid leukemia and RAS mutations benefit most from postremission high-dose cytarabine: a Cancer and Leukemia Group B study. Clin Oncol 26: 4603-4609.

52. Kiessling MK, Oberholzer PA, Mondal C, Karpova MB, Zipser MC et al. (2011) High-throughput mutation profiling of CTCL samples reveals KRAS and NRAS mutations sensitizing tumors toward inhibition of the RAS/RAF/MEK signaling cascade. Blood 117: 2433-2440.

53. Renneville A, Roumier C, Biggio V, Nibourel O, Boissel N, et al. (2008) Cooperating gene mutations in acute myeloid leukemia: a review of the literature. Leukemia 22: 915-931.

54. Langer C, Radmacher MD, Ruppert AS, Whitman SP, Paschka P, et al. (2008) High BAALC expression associates with other molecular prognostic markers, poor outcome, and a distinct gene-expression signature in cytogenetically normal patients younger than 60 years with acute myeloid leukemia: A Cance and Leukemia Group B (CALGB) study. Blood 111:5371-5379.

55. Langer C, Marcucci G, Holland KB, Radmacher MD, Maharry K, et al. (2009) Prognostic importance of MN1 transcript levels, and biologic insights from MN1-associated gene and microRNA expression signatures in cytogenetically normal acute myeloid leukemia: A Cancer and Leukemia Group B study. J Clin Oncol 27:3198-3204

56. Marcucci G, Maharry K, Whitman SP, Vukosavljevic T, Paschka P, et al. (2007) High expression levels of the ETS-related gene, ERG, predict adverse outcome and improve molecular risk-based classification of cytogenetically normal acute myeloid leukemia: a Cancer and Leukemia Group B Study. J Clin Oncol 25 3337-3343.

57. Gröschel S, Lugthart S, Schlenk RF, Valk PJ, Eiwen K, et al. (2010) High EVI1 expression predicts outcome in younger adult patients with acute myeloid leukemia and is associated with distinct cytogenetic abnormalities. $\mathrm{J}$ Clin Oncol 28: 2101-2107.

58. Dolnik A, Engelmann JC, Scharfenberger-Schmeer M, Mauch J, Kelkenberg Schade S, et al. (2012) Commonly altered genomic regions in acute myeloid leukemia are enriched for somatic mutations involved in chromatin remodeling and splicing. Blood 120: e83-92.

59. Varambally S, Dhanasekaran SM, Zhou M, Barrette TR, Kumar-Sinha C, et al. (2002) The polycomb group protein EZH2 is involved in progression of prostate cancer. Nature 419: 624-629.

60. Benetatos L, Voulgaris E, Vartholomatos G, Hatzimichael E (2013) Noncoding RNAs and EZH2 interactions in cancer: Long and short tales from the transcriptome. Int J Cancer 133: 267-274

61. Tan J, Yang X, Zhuang L, Jiang X, Chen W, et al. (2007) Pharmacologic disruption of Polycomb-repressive complex 2-mediated gene repression selectively induces apoptosis in cancer cells. Genes Dev 21: 1050-1063.

62. Zhou J, Bi C, Cheong LL, Mahara S, Liu SC, et al. (2011) The histone methyltransferase inhibitor, DZNep, up-regulates TXNIP, increases ROS production, and targets leukemia cells in AML. Blood 118: 2830-2839. 
Citation: Sharma M, Mohanty S (2013) Molecular Biomarkers in Cytogenetically Normal -Acute Myeloid Leukemia: harnessing the targets. J Mo Biomarkers Diagn S8:009. doi:10.4172/2155-9929.S8-009

63. Fiskus W, Wang Y, Sreekumar A, Buckley KM, Shi H, et al. (2009) Combined epigenetic therapy with the histone methyltransferase EZH2 inhibitor 3-deazaneplanocin $\mathrm{A}$ and the histone deacetylase inhibitor panobinostat against human AML cells. Blood 114: 2733-2743.

64. Schwind S, Maharry K, Radmacher MD, Mrózek K, Holland KB, et al. (2010) Prognostic significance of expression of a single microRNA, miR-181a, in cytogenetically normal acute myeloid leukemia: a Cancer and Leukemia Group B study. J Clin Oncol 28: 5257-5264.

65. Garzon R, Volinia S, Liu CG, Fernandez-Cymering C, Palumbo T, et al. (2008) MicroRNA signatures associated with cytogenetics and prognosis in acute myeloid leukemia. Blood 111: 3183-3189.

66. Xu Q, Simpson SE, Scialla TJ, Bagg A, Carroll M (2003) Survival of acute myeloid leukemia cells requires PI3 kinase activation. Blood 102: 972-980.

67. Kornblau SM, Womble M, Qu YH, Jackson CE, Chen W, et al. (2006) Simultaneous activation of multiple signal transduction pathways confers poor prognosis in acute myelogenous leukemia. Blood 108: 2358-2365.

68. Kojima K, Shimanuki M, Shikami M, Samudio IJ, Ruvolo V, et al. (2008) The dual $\mathrm{PI} 3$ kinase/mTOR inhibitor $\mathrm{PI}-103$ prevents $\mathrm{p} 53$ induction by $\mathrm{Mdm} 2$ inhibition but enhances p53-mediated mitochondrial apoptosis in p53 wild-type AML. Leukemia 22: 1728-1736.

69. Park S, Chapuis N, Bardet V, Tamburini J, Gallay N, et al. (2008) PI-103, a dual inhibitor of Class IA phosphatidylinositide 3-kinase and mTOR, has antileukemic activity in AML. Leukemia 22: 1698-1706.

70. Alam MM, Joh EH, Kim Y, Oh YI, Hong J, et al. (2012) Synthesis and biologica evaluation of cyclopentane-linked alkyl phosphocholines as potential anticance agents that act by inhibiting Akt phosphorylation. Eur J Med Chem 47: 485-492.

71. Rahmani M, Anderson A, Habibi JR, Crabtree TR, Mayo M, et al. (2009) The $\mathrm{BH} 3-$ only protein Bim plays a critical role in leukemia cell death triggered by concomitant inhibition of the PI3K/Akt and MEK/ERK1/2 pathways. Blood 114 4507-4516.

72. Yee KW, Zeng Z, Konopleva M, Verstovsek S, Ravandi F, et al. (2006) Phase I/II study of the mammalian target of rapamycin inhibitor everolimus (RAD001) in patients with relapsed or refractory hematologic malignancies. Clin Cancer Res 12: 5165-5173.

73. Xu Q, Thompson JE, Carroll M (2005) mTOR regulates cell survival after etoposide treatment in primary AML cells. Blood 106: 4261-4268.

74. Chapuis N, Tamburini J, Green AS, Vignon C, Bardet V, et al. (2010) Dua inhibition of PI3K and mTORC1/2 signaling by NVP-BEZ235 as a new therapeutic strategy for acute myeloid leukemia. Clin Cancer Res 16: 54245435.

75. Cortes JE, Feldman EJ, Yee K, Rizzieri DA, Advani AS, et al. (2011) Results of the OPAL Study: A Phase II Study to Evaluate the Efficacy, Safety and Tolerability of Tosedostat (CHR-2797) in Elderly Subjects with Treatment Refractory or Relapsed Acute Myeloid Leukemia. Blood

76. Amadori S, Stasi R, Martelli AM, Venditti A, Meloni G, et al. (2012) Temsirolimus, an mTOR inhibitor, in combination with lower-dose clofarabine as salvage therapy for older patients with acute myeloid leukaemia: results of a phase II GIMEMA study (AML-1107). Br J Haematol 156: 205-212.

77. Levis M, Ravandi F, Wang ES, Baer MR, Perl A, et al. (2011) Results from a randomized trial of salvage chemotherapy followed by lestaurtinib for patients with FLT3 mutant AML in first relapse. Blood 117: 3294-3301.

78. Schnittger S, Kern W, Tschulik C, Weiss T, Dicker F, et al. (2009) Minima residual disease levels assessed by NPM1 mutation-specific RQ-PCR provide important prognostic information in AML. Blood 114: 2220-2231.

79. Kim DH, Lee NY, Sung WJ, Baek JH, Kim JG, et al. (2005) Multidrug resistance as a potential prognostic indicator in acute myeloid leukemia with normal karyotypes. Acta Haematol 114: 78-83.

80. Sievers EL, Smith FO, Woods WG, Lee JW, Bleyer WA, et al. (1995) Cell surface expression of the multidrug resistance P-glycoprotein (P-170) as detected by monoclonal antibody MRK-16 in pediatric acute myeloid leukemia fails to define a poor prognostic group: A report from the Children's Cancer Group. Leukemia 9:2042-2048.

81. Willman CL (1997) The prognostic significance of the expression and function of multidrug resistance transporter proteins in acute myeloid leukemia: Studies of the Southwest Oncology Group Leukemia Research Program. Semin Hematol 34: 25-33
82. Campos L, Guyotat D, Archimbaud E, Calmard-Oriol P, Tsuruo T, et al. (1992) Clinical significance of multidrug resistance $\mathrm{P}$-glycoprotein expression on acute nonlymphoblastic leukemia cells at diagnosis. Blood 79: 473-476.

83. Del Poeta G, Stasi R, Aronica G, Venditti A, Cox MC, et al. (1996) Clinical relevance of $\mathrm{P}$-glycoprotein expression in de novo acute myeloid leukemia. Blood 87: 1997-2004.

84. Leith CP, Kopecky KJ, Godwin J, McConnell T, Slovak ML, et al. (1997) Acute myeloid leukemia in the elderly: Assessment of multidrug resistance (MDR1) and cytogenetics distinguishes biologic subgroups with remarkably distinct responses to standard chemotherapy. A Southwest Oncology Group study. Blood 89:3323-3329.

85. Leith CP, Kopecky KJ, Chen IM, Eijdems L, Slovak ML, et al. (1999) Frequency and clinical significance of the expression of the multidrug resistance proteins MDR1/P-glycoprotein, MRP1, and LRP in acute myeloid leukemia: a Southwest Oncology Group Study. Blood 94: 1086-1099.

86. Matsui H, Takeshita A, Naito K, Shinjo K, Shigeno K, et al. (2002) Reduced effect of gemtuzumab ozogamicin (CMA-676) on P-glycoprotein and/or CD34positive leukemia cells and its restoration by multidrug resistance modifiers. Leukemia 16: 813-819.

87. Linenberger ML, Hong T, Flowers D, Sievers EL, Gooley TA, et al. (2001) Multidrug-resistance phenotype and clinical responses to gemtuzumab ozogamicin. Blood 98: 988-994.

88. Ross DD (2004) Modulation of drug resistance transporters as a strategy for treating myelodysplastic syndrome. Best Pract Res Clin Haematol 17: 641-651.

89. Meshinchi S, Arceci RJ (2007) Prognostic factors and risk-based therapy in pediatric acute myeloid leukemia. Oncologist 12: 341-355.

90. Blum W, Marcucci G (2008) New approaches in acute myeloid leukemia. Best Pract Res Clin Haematol 21: 29-41.

91. Tallman MS (2005) New strategies for the treatment of acute myeloid leukemia including antibodies and other novel agents. Hematology Am Soc Hematol Educ Program.

92. Fathi AT, Grant S, Karp JE (2010) Exploiting cellular pathways to develop new treatment strategies for AML. Cancer Treat Rev 36: 142-150.

93. Lehmann S, Bykov VJ, Ali D, Andrén O, Cherif H, et al. (2012) Targeting p53 in vivo: a first-in-human study with p53-targeting compound APR-246 in refractory hematologic malignancies and prostate cancer. J Clin Oncol 30: 3633-3639.

94. Tani T, Ylänne J, Virtanen I (1996) Expression of megakaryocytic and erythroid properties in human leukemic cells. Exp Hematol 24: 158-168.

95. Blair A, Pamphilon DH (2003) Leukaemic stem cells. Transfus Med 13: 363 375

96. Ravandi F, Estrov Z (2006) Eradication of leukemia stem cells as a new goal of therapy in leukemia. Clin Cancer Res 12: 340-344.

97. Guzman ML, Rossi RM, Karnischky L, Li X, Peterson DR, et al. (2005) The sesquiterpene lactone parthenolide induces apoptosis of human acute myelogenous leukemia stem and progenitor cells. Blood 105:4163-9.

98. Xu Q, Simpson SE, Scialla TJ, Bagg A, Carroll M (2003) Survival of acute myeloid leukemia cells requires PI3 kinase activation. Blood 102: 972-980.

99. Schliemann C, Bieker R, Thoennissen N, Gerss J, Liersch R, et al. (2007) Circulating angiopoietin-2 is a strong prognostic factor in acute myeloid leukemia. Leukemia 21: 1901-1906.

100. Petit I, Karajannis MA, Vincent L, Young L, Butler J, et al. (2008) The microtubule-targeting agent CA4P regresses leukemic xenografts by disrupting interaction with vascular cells and mitochondrial-dependent cell death. Blood 111: 1951-1961.

101. Gorin NC, Estey E, Jones RJ, Levitsky HI, Borrello I, et al. (2000) New Developments in the Therapy of Acute Myelocytic Leukemia. Hematology Am Soc Hematol Educ Program.

102. Mikkola HK, Radu CG, Witte ON (2010) Targeting leukemia stem cells. Nat Biotechnol 28: 237-238

103. Yamazaki Y, Kunimoto S, Ikeda D (2007) Rakicidin A: a hypoxia-selective cytotoxin. Biol Pharm Bull 30: 261-265.

104. Kottaridis PD, Gale RE, Langabeer SE, Frew ME, Bowen DT, et al. (2002) Studies of FLT3 mutations in paired presentation and relapse samples from patients with acute myeloid leukemia: implications for the role of FLT3 
Citation: Sharma M, Mohanty S (2013) Molecular Biomarkers in Cytogenetically Normal -Acute Myeloid Leukemia: harnessing the targets. J Mol Biomarkers Diagn S8:009. doi:10.4172/2155-9929.S8-009

Page 9 of 9

mutations in leukemogenesis, minimal residual disease detection, and possible therapy with FLT3 inhibitors. Blood 100(7):2393-8.

105. List AF, Kopecky KJ, Willman CL, Head DR, Persons DL, et al. (2001) Benefit of cyclosporine modulation of drug resistance in patients with poor-risk acute myeloid leukemia: a Southwest Oncology Group study. Blood 98: 3212-3220.

106. Baer MR, George SL, Dodge RK, O'Loughlin KL, Minderman H, et al. (2002) Phase 3 study of the multidrug resistance modulator PSC-833 in previously untreated patients 60 years of age and older with acute myeloid leukemia: Cancer and Leukemia Group B Study 9720. Blood 100: 1224-1232.

107.Plasschaert SL, Van Der Kolk DM, De Bont ES, Vellenga E, Kamps WA, et al. (2004) Breast cancer resistance protein (BCRP) in acute leukemia. Leuk Lymphoma 45: 649-654.

108. Fracasso PM, Goldstein LJ, de Alwis DP, Rader JS, Arquette MA, et al. (2004) Phase I study of docetaxel in combination with the P-glycoprotein inhibitor, zosuquidar, in resistant malignancies. Clin Cancer Res 10: 7220-7228.

109.Fang K, Qian F, Chen YQ (2012) MicroRNAs as regulators in norma hematopoietic and leukemia stem cells: current concepts and clinical implications. Curr Mol Med 12: 536-546.
110. Chumsri S, Matsui W, Burger AM (2007) Therapeutic implications of leukemic stem cell pathways. Clin Cancer Res 13: 6549-6554.

111. Welch JS, Link DC (2011) Genomics of AML: clinical applications of nextgeneration sequencing. Hematology Am Soc Hematol Educ Program 2011: 30-35.

112. Hartford CM, Duan S, Delaney SM, Mi S, Kistner EO, et al. (2009) Populationspecific genetic variants important in susceptibility to cytarabine arabinoside cytotoxicity. Blood 113: 2145-2153.

113. Gulley ML, Shea TC, Fedoriw Y (2010) Genetic tests to evaluate prognosis and predict therapeutic response in acute myeloid leukemia. J Mol Diagn 12: 3-16.

114. Figueroa ME, Lugthart S, Li Y, Erpelinck-Verschueren C, Deng X, et al. (2010) DNA methylation signatures identify biologically distinct subtypes in acute myeloid leukemia. Cancer Cell 17: 13-27.

115. Shen Y, Zhu YM, Fan X, Shi JY, Wang QR, et al. (2011) Gene mutation patterns and their prognostic impact in a cohort of 1185 patients with acute myeloid leukemia. Blood 118: 5593-5603.
This article was originally published in a special issue, Potential Biomarkers and Therapeutic Targets in Cancer Stem Cells handled by Editor(s). Dr. Murielle Mimeault, University of Nebraska Medical Center, USA 